\title{
Preschool vision screening: a recent report calls for a halt
}

Purchasers are advised against implementing preschool vision screening programmes and providers should consider discontinuing them. This is the clearly stated recommendation of a recent report by the NHS Centre for Reviews and Dissemination (NHS CRD). ${ }^{1}$ The role of this organisation is to promote best practice by disseminating its findings to key decision makers in the NHS and to consumers of healthcare services. Hence, calls to consider and implement the report's findings are likely to be swift.

The report was prepared by the Health Services Research Unit at Oxford University backed up by an advisory group which included representatives from all the ophthalmic professions. The bulk of the report is given over to a systematic review of research on the effectiveness of preschool vision screening (PSVS). An additional remit was to provide evidence on which decisions about future provisions of service can be based, and to highlight areas where future research is required. The review was restricted to studies involving the target conditions of amblyopia, refractive error, and non-cosmetically obvious squint (NCOS). In addition to hand searching, 17 electronic databases were consulted initially flagging over 5000 references. More than 500 abstracts were scrutinised and 85 publications included in the main analysis. Research questions of relevance to the objectives were posed under the headings of prevalence, natural history, disability, treatment, and screening.

\section{Prevalence}

No studies were identified whose primary aim was to establish the prevalence of the target conditions. Prevalence estimates ranging from 2.4 to $6.1 \%$ (all target condition combined) were sourced from retrospective analyses of hospital records and from observational studies of the yield from screening programmes. On the basis of these figures, it was concluded that the target conditions were sufficiently prevalent to justify a consideration for screening.

\section{Natural history}

The reviewers were unable to locate any studies designed with the intention of documenting natural history although some background data were gleaned from studies which did not meet the review's strict inclusion criteria. The report notes that without a clear picture of natural history, one cannot appraise treatment effectiveness in the absence of a no treatment control group.

The report makes several references to the possibility that amblyopia may, under certain circumstances, undergo spontaneous remission. While this phenomenon has been reported in patients who subsequently suffer a loss of vision in their fellow eye ${ }^{2}$ it is not generally thought to occur in the natural course of events. In one study, repeatedly cited in the report, ${ }^{3}$ in which evidence for spontaneous remission was found, the investigators' alternative explanation is not acknowledged.

\section{Disability}

The search for studies on disability proved more productive. Twenty one relevant studies were identified covering five different study designs. None the less, the reviewers were unable to find evidence sufficient to "...offer any advice to parents if the target conditions are left untreated." No evidence could be found to substantiate claims that either refractive errors or NCOS were disabling.

\section{Treatment}

Evidence to support the effectiveness of orthoptic treatment (including occlusion therapy) was deemed "disappointing" but did however "substantiate clinical beliefs that children with amblyopia do improve during treatment." However, in referring back to the lack of evidence of the natural history of the target conditions, the reviewers stress that the evidence "...falls very far short of showing that treatment works."

Subsequent commentators ${ }^{4}$ have pointed out that, for the most part, the evidence underpinning amblyopia treatment stems from animal, rather than from human, studies which were not appraised by the report. It is the former body of work by, among others, the Nobel laureates Hubel and Wiesel, which exerted a powerful influence on clinical practice and largely explains why treatment trials, particularly those with a "no treatment arm," have not been undertaken. None the less, it should be borne in mind that much of the animal literature relates to amblyopia arising from stimulus deprivation - a condition whose pathophysiology may differ substantially from that of the target conditions.

\section{Screening}

Studies of orthoptic screening provided "reasonable" evidence that it could be undertaken in the UK with acceptable uptake and referral rates. Only one prospective controlled trial was identified comparing differing screening protocols (principally in terms of the professions involved). Primary orthoptic screening was found to be superior to that provided by other professional groups in detecting amblyopia and refractive errors (but not necessarily squint) but did not support the conclusion that the prevalence of amblyopia was reduced at 7 years of age.

\section{Comment}

The conclusions of the report are acknowledged to differ from those previously published which have judged PSVS to be worthwhile..$^{5-7}$ This is attributed to a more rigorous approach in reviewing disability and treatment studies undertaken by the present report.

Given its findings, it is perhaps unsurprising that the report's recommendations are forthrightly expressednamely, that new screening programmes should not be implemented and that current programmes should only be continued in the context of a controlled trial of treatment. The forcefulness of this statement is backed up by the assertion that "the ethical basis for such interventions (that is, screening) is very insecure" (my parenthesis).

Is it unethical to screen in the absence of good evidence of its effectiveness? All but the most evangelistic proponents of evidence based medicine would argue that a lack of evidence of effectiveness is not evidence of ineffectiveness; in a routine clinical setting this permits the treatment of patients according to prevailing clinical wisdom even 
where rigorous proof of both treatment effectiveness and of a significant risk of disablement is lacking. However, others claim that when screening is considered, a "higher burden of proof" is required such that, in contrast with the routine clinic setting, no evidence of effectiveness is sufficient evidence of ineffectiveness. ${ }^{8}$ The argument runs that when undertaking screening one is making the implicit assumption that screening will benefit the child and that this needs to be firmly established before screening can be justified. Screening programmes carry inherent risks ${ }^{9}$; in the present case, orthoptic treatment and spectacle wear have the potential to adversely influence the child's psychological wellbeing. ${ }^{10}$ Further, if amblyopia cannot be shown to be disabling how can it be possible to obtain informed consent for treatment? Critics of this position would claim that evidence for actual harm being caused is far from firmly established and that it is self evident that a reduction in visual acuity and stereopsis must be disabling - certainly there are occupational consequences.

Perhaps in anticipation of protestations, the report's authors have latterly addressed the consequences of scrapping PSVS. ${ }^{11}$ Here, they acknowledge the likely detrimental effect on the orthoptic profession, and on parents and teachers who, for many years, have been advised of the importance of screening. They also point out that once scrapped, PSVS could not be rapidly re-established. As a solution to this dilemma they suggest that parents should be provided with written information addressing the shortcomings of our knowledge as highlighted in the report. It might be argued that were this to accurately reflect the report's conclusions, albeit in lay language, the uptake to any programme might be so small as to render it unviable.

If those who have invested time and effort in promoting or undertaking PSVS feel that the rug has been pulled from under their feet, those with a research agenda relating to the target conditions should be buoyed up. Recommendations for future research included in the report are wide ranging with key questions being posed under all topics covered by the review. Even here though the report courts controversy; a proposed investigation into the effectiveness of orthoptic treatment calls specifically for a randomised trial involving a no treatment control group-something that many clinical investigators, not to mention research ethics committees, may balk at.

I wish to thank Dr Sarah-Stewart Brown and Professor Alistair Fielder for their helpful discussion during the preparation of this article. The author was a member of the report's advisory group which did not have responsibility for its content.

\section{MERRICK J MOSELEY}

Imperial College of Science, Technology and Medicine, Academic Unit of Ophthalmology, Western Eye Hospital, Marylebone Road, London NW1 5 YE

1 Snowden SK, Stewart-Brown SL. Preschool vision screening: results of a systematic review. Report No 9. York: NHS Centre for Reviews and Dissemination, University of York.

2 Vereecken EP, Brabant P. Prognosis for vision in amblyopia after the loss of the good eye. Arch Ophthalmol 1984;102:220-4.

3 Bray LC, Clarke MP, Jarvis SN, et al. Preschool vision screening: a prospective evaluation. Eye 1996;10:714-8.

4 Rahi JS, Dezateux C. The future of preschool vision screening services in Britain. BMF 1997;315:1247-8.

5 Guide to Clinical Preventative Services. Report of the US Services Preventative Task Force. 2nd ed. Baltimore: Williams and Wilkins, 1994:373-82.

6 Canadian Task Force on the Periodic Health Examination. The Canadian Guide to Clinical Preventative Health Care. Ottawa: Canada Communication Group, 1994:298-304.

7 Simons K. Preschool vision screening: rationale, methodology and outcome. Surv Ophthalmol 1996;41:3-30.

8 Stewart-Brown SL. Evaluating screening programmes: theory and practice. In: Jenkinson C, ed. Assessment and evaluation of health and medical care. Open University Press.

9 Mant D, Fowler G. Mass screening: theory and ethics. BMF 1990;300:916-8. 10 Snowden SK, Stewart-Brown SL. Amblyopia and disability: a qualitative study. Oxford: Health Services Research Unit, University of Oxford, 1997.

11 Stewart-Brown SL, Snowden SK. Evidence-based dilemmas in preschool vision screening. Arch Dis Childh (in press). 